\title{
Identitätsbasierte Markenführung
}


Christoph Burmann - Tilo Halaszovich Frank Hemmann

\section{Identitätsbasierte Markenführung}

Grundlagen - Strategie Umsetzung - Controlling

Mit einem Beitrag von

Prof. Dr. Dr. h.c. mult. Heribert Meffert

Springer Gabler 
Prof. Dr. Christoph Burmann

Dr. Tilo Halaszovich

Frank Hemmann

Lehrstuhl für innovatives Markenmanagement

Universität Bremen, Deutschland

Die Deutsche Nationalbibliothek verzeichnet diese Publikation in der Deutschen Nationalbibliografie; detaillierte bibliografische Daten sind im Internet über http://dnb.d-nb.de abrufbar.

\section{Springer Gabler}

(C) Springer Fachmedien Wiesbaden 2012

Das Werk einschließlich aller seiner Teile ist urheberrechtlich geschützt. Jede Verwertung, die nicht ausdrücklich vom Urheberrechtsgesetz zugelassen ist, bedarf der vorherigen Zustimmung des Verlags. Das gilt insbesondere für Vervielfältigungen, Bearbeitungen, Übersetzungen, Mikroverfilmungen und die Einspeicherung und Verarbeitung in elektronischen Systemen.

Die Wiedergabe von Gebrauchsnamen, Handelsnamen, Warenbezeichnungen usw. in diesem Werk berechtigt auch ohne besondere Kennzeichnung nicht zu der Annahme, dass solche Namen im Sinne der Warenzeichen- und Markenschutz-Gesetzgebung als frei zu betrachten wären und daher von jedermann benutzt werden dürften.

Lektorat: Barbara Roscher, Jutta Hinrichsen

Gedruckt auf säurefreiem und chlorfrei gebleichtem Papier

Springer Gabler ist eine Marke von Springer DE. Springer DE ist Teil der Fachverlagsgruppe Springer Science+Business Media.

www.springer-gabler.de 


\section{Vorwort}

Marken sind für den Erfolg von Unternehmen von größter Relevanz. Deswegen gibt es heute eine Fülle wissenschaftlicher und praxisorientierter Publikationen über Marken und deren richtige Führung. Das Konzept der identitätsbasierten Markenführung hat sich dabei als das leistungsfähigste Managementmodell erwiesen. Die Basis für dieses Konzept wurde Mitte der 1990er Jahre von David Aaker in den USA, Jean-Noel Kapferer in Frankreich sowie Heribert Meffert und Christoph Burmann in Deutschland entwickelt. Die identitätsbasierte Markenführung ergänzt die externe Sicht auf die Marke (z.B. seitens der Kunden, Absatzmittler und Wettbewerber) um die interne Sicht des Managements und der Mitarbeitern einer Marke. Der Wirkungsanalyse von Marken in Märkten wird damit eine Analyse des Führungsverhaltens (brand leadership) sowie der internen Strukturen und Prozesse der Marken gegenübergestellt. Als theoretisches Fundament greift die identitätsbasierte Markenführung auf die „competence based theory of the firm“ zurück, den aktuell wichtigsten Erklärungsansatz im Rahmen der strategischen Managementforschung.

Beim Einsatz der identitätsbasierten Markenführung in der Aus- und Weiterbildung zeigte sich bisher ein wichtiges Defizit: Studenten und Praktiker fanden kein geeignetes Lehrbuch, in dem das Konzept vollständig, aktuell und vor allem kompakt erläutert wurde. Diese Lücke wollen wir mit unserem Buch schließen. Es soll einen leicht verständlichen Überblick geben und zeigen, dass dieser Markenführungsansatz praktisch anwendbar ist und großes Erfolgspotenzial besitzt. Um den praktischen Nutzen der identitätsbasierten Markenführung zu belegen, haben wir zahlreiche Beispiele in das Buch integriert. Für die uns hierbei gewährte Unterstützung vieler Unternehmen möchten wir uns an dieser Stelle bedanken.

Die Erstellung dieses Buches wäre ohne die Unterstützung zahlreicher Personen kaum möglich gewesen. Zunächst ist Univ.-Prof. Dr. Dr. h.c. mult. Heribert Meffert zu erwähnen, der unser Konzept der identitätsbasierten Markenführung in den Anfängen wesentlich mitgeprägt hat. Unser Dank gilt ferner den wissenschaftlichen Mitarbeitern am LiM der Universität Bremen, namentlich besonders Frau Barbara Kleine-Kalmer, Herrn Dr. Andreas Müller, Frau Antje Löwa, Herrn Behzad Zabeti und Herrn Dr. Michael Schade. Darüber hinaus haben die studentischen Mitarbeiter am LiM ganz erheblich zum Gelingen dieses Buches beigetragen. Hier sind besonders zu erwähnen Frau Corinna Beckmann, Herr Sacid Celik, Herr Stephan Hanisch, Frau Natalie Skutzik, Herr Daniel Segelken und Herr Dominik Middeke. Wir danken auch Frau Angela Pfeiffer von Springer Gabler für ihren großen Einsatz beim Satz und der Gestaltung dieses Buches. Abschließend möchten wir uns auch bei Frau Barbara Roscher von Springer Gabler bedanken, die uns - wie immer - sehr gut betreut hat.

Zur Vertiefung des Konzeptes der identitätsbasierten Markenführung stehen mittlerweile über 50 erfolgreich abgeschlossene Dissertationen zur Verfügung, die an der Universität Bremen (am Lehrstuhl für innovatives Markenmanagement) und an der Handelshochschule in Leipzig (am Lehrstuhl für Marketingmanagement) entstanden sind. Sie wurden fast alle in der Buchreihe „Innovatives Markenmanagement“ bei Springer Gabler publiziert. 
Wir wünschen Ihnen nun viele interessante, neue Einsichten beim Lesen und freuen uns auf das Feedback unserer Leser.

Bremen

Univ.-Prof. Dr. Christoph Burmann

Dr. Tilo Halaszovich

Dipl.-Kfm. Frank Hemmann 


\section{Inhalt}

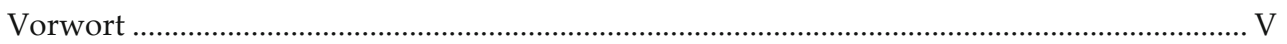

$1 \quad$ Grundlagen der identitätsbasierten Markenführung ….......................................... 1

2 Das Konzept der identitätsbasierten Markenführung ............................................ 19

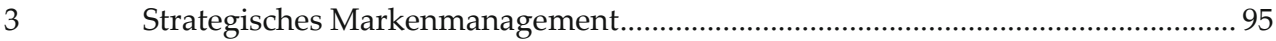

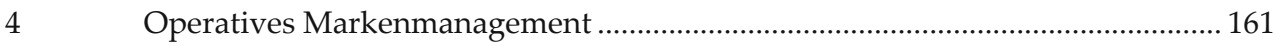

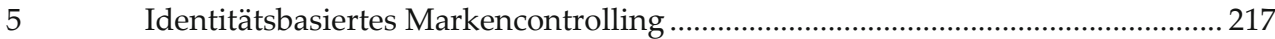

6 Markenführung im Wandel - alte Weisheiten und neue Erkenntnisse................. 269

Prof. Dr. Dr. mult. h.c. Heribert Meffert

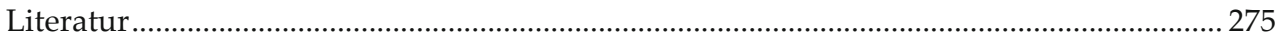

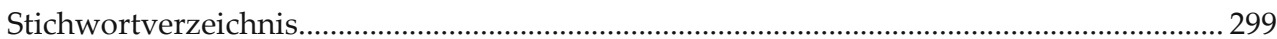

